\title{
A FORMAÇÃO DE COMUNIDADES-FILHAS NO FLUXO DE BRASILEIROS PARA OS ESTADOS UNIDOS
}

\author{
Wilson Fusco
}

\begin{abstract}
Resumo: A proposta deste artigo é analisar a formação de comunidades brasileiras nos Estados Unidos como resultado do processo de organização social que facilita a adaptação do imigrante no destino. Fortemente influenciados pelas redes migratórias, os migrantes em potencial deixam de considerar muitas localidades teoricamente disponíveis e concentram-se naqueles poucos locais com os quais seu lugar de origem possui laços sociais importantes.

Palavras-chave: Migração internacional. Estados Unidos. Rede social.

Abstract: The purpose of this work is to analyze the formation of Brazilian communities in certain areas of the U.S.A. as a result of the social organization process which facilitates the immigrant adjustment at his/her destination. Strongly influenced by migrant networks, the potential migrants fail to consider many available places as potential destinations in order to concentrate themselves at those few places, which are connected to their place of origin through relevant social ties.

Key words: International Migration. United States. Social Network.
\end{abstract}

$\Lambda$ formação de comunidades de imigrantes nos Estados Unidos é um processo amplamente registrado na literatura especializada. Massey (1987) é o único autor a usar o termo "daughter communities" (comunidades-filhas) para descrever o caso dos mexicanos na Califórnia, mas vários outros estudiosos que analisam processos semelhantes podem ser citados, como Portes (2002), que encontra "enclaves" de imigrantes sul-americanos em Los Angeles, Nova York e Washington, ou Menjivar (1997), que analisa os salvadorenhos em San Francisco. Margolis (1994), que estudou os brasileiros em Nova York, descreve uma comunidade "invisível", principalmente em função da ausência de instituições associativas direcionadas aos brasileiros.

O conceito de "rede social" na migração internacional é entendido por Massey (1987) como um conjunto de la- ços sociais que ligam comunidades de origem a pontos de destino específicos. Tais laços unem migrantes e nãomigrantes em uma complexa teia de papéis sociais complementares e de relacionamentos interpessoais que são mantidos por um quadro informal de expectativas mútuas e comportamentos predeterminados.

A origem dos fluxos migratórios confunde-se com a expansão de determinadas redes sociais. Estas se consolidam e formam vias transmissoras de recursos necessários para a adaptação do migrante no destino, ao mesmo tempo em que, na origem, estimulam o migrante em potencial a ingressar no fluxo.

Em consonância com a argumentação de Douglas Massey acerca da formação de comunidades-filhas entre mexicanos, este trabalho pretende mostrar algumas características da organização social própria do processo de 
concentração de emigrantes brasileiros em regiões específicas nos Estados Unidos. A hipótese a ser explorada é a de que a formação de comunidades de brasileiros em determinadas áreas nos EUA pode ser explicada, para além dos fatores econômicos, em função da utilização do capital social, cujos benefícios circulam por meio das redes sociais, principalmente redes de parentesco e amizade, que alimentam e regulam os fluxos continuamente.

\section{BASE DE DADOS}

Os dados utilizados para subsidiar este trabalho são provenientes de dois surveys - um, realizado em Governador Valadares-MG, e o outro, em Criciúma-SC. O survey realizado em Governador Valadares-MG, finalizado em julho de 1997, fez parte do projeto CNPq (MCT/Finep/ Pronex) intitulado "Imigrantes Brasileiros nos EUA Cidadania e Identidade"; o realizado em Criciúma-SC, levado a termo em junho de 2001, foi financiado pelo projeto Fapesp "As redes sociais nas migrações internacionais: os migrantes brasileiros para os Estados Unidos e Japão". Ambos os projetos foram coordenados pela Professora Dra. Teresa Sales e desenvolvidos no Núcleo de Estudos de População - Nepo da Unicamp.

O método utilizado para o planejamento das pesquisas de campo pode ser descrito como "amostragem aleatória por conglomerados simples". Esse processo permite inferir os resultados obtidos à população total, uma vez que todas as combinações de " $\mathrm{n}$ " unidades entre os "N" indivíduos da população têm a mesma probabilidade de pertencer à amostra, com erro e intervalo de confiança controlados. ${ }^{1}$ Dessa forma, podemos extrapolar os resultados das amostras e dizer que as cidades de Governador Valadares e Criciúma têm, no mínimo, 6,7\% e 3,2\% de suas populações com experiência migratória internacional, respectivamente. Uma vez encontrados os domicílios com migrantes internacionais, foram aplicados questionários para capturar as características sociodemográficas da população envolvida com o processo, juntamente com diversos aspectos do histórico migratório de cada indivíduo.

A principal vantagem de surveys como os que foram utilizados para este trabalho é a de suprir a carência sistemática de dados oficiais representativos sobre a migração internacional de brasileiros. As fontes secundárias de dados não são capazes de captar as informações necessárias para as análises que os estudiosos da migração se propõem a elaborar - e isso acontece principalmente em fun- ção da ilegalidade dos migrantes. Além disso, um estudo especialmente valioso para este trabalho é a pesquisa de campo realizada com a intenção explícita de captar elementos particulares da organização social dos migrantes, e que permita a comparação entre diferentes locais de origem.

Também cabe reconhecer um fator negativo das fontes de dados utilizadas. Por terem sido realizados somente em uma das "pontas" do fluxo migratório, esses estudos carregam um limite importante: algumas das informações não puderam ser obtidas diretamente dos migrantes, pois, no momento das entrevistas, estes estavam vivendo nos Estados Unidos. Na verdade, tais informações foram dadas pelos migrantes retornados ou por parentes em condições de fazê-lo.

\section{CONCENTRAÇÃO NO DESTINO}

A rota que leva aos Estados Unidos é a principal escolha dos emigrantes brasileiros. Segundo estimativa divulgada pelo Ministério das Relações Exteriores para 2001 (Tabela 1), aproximadamente $42 \%$ dos brasileiros que viviam no exterior estavam nos Estados Unidos, 23\% estavam no Paraguai e $12 \%$ no Japão. Os emigrantes para Paraguai e Japão guardam características específicas aos respectivos movimentos: os primeiros têm origem na região do entorno da fronteira com o Paraguai e fixam-se na zona rural (PALAU, 2001); os dekasseguis, ${ }^{2}$ por sua vez, são cidadãos brasileiros que se enquadram nas exigências legais relativas à ascendência étnica para a obtenção do visto apropriado, concedido pelo governo japonês (SASAKI, 1999).

Os brasileiros que decidem viver nos Estados Unidos carregam a marca da ilegalidade: ao mesmo tempo em que o Ministério das Relações Exteriores calcula em 800 mil os brasileiros que estão morando nos Estados Unidos, a

TABELA 1

Migrantes Brasileiros, segundo País de Residência Brasil - 2001

\begin{tabular}{lcc}
\hline País de Residência & População & $\%$ \\
\hline Total & 1.887 .895 & 100,00 \\
Estados Unidos & 799.203 & 42,33 \\
Paraguai & 442.104 & 23,42 \\
Japão & 224.970 & 11,92 \\
Outros & 421.618 & 22,33 \\
\hline
\end{tabular}

Fonte: Ministério das Relações Exteriores (2001). 
estimativa do Bureau of the Census deste país coloca esse número entre 160 e 230 mil brasileiros (UNITED STATES BUREAU OF THE CENSUS, 2000, Tabelas 3-4). É notório que o registro de brasileiros é feito somente para aqueles que têm a situação de imigrante regularizada, visto que os indocumentados não se declaram, pelo risco de prisão e deportação. O que se conclui é que a maioria dos migrantes vive de forma clandestina nos Estados Unidos.

Os Estados Unidos também atraem a maioria dos emigrantes de Governador Valadares e de Criciúma. Segundo a Tabela 2, mais de $85 \%$ dos valadarenses e $60 \%$ dos criciumenses escolheram os EUA como destino em sua primeira viagem ao exterior. As proporções de migrantes com experiência nos EUA sobem respectivamente para $88,7 \%$ e $65,4 \%$, quando são considerados também os indivíduos que, tendo inicialmente escolhido outro país em sua primeira viagem, decidem-se pelos Estados Unidos num momento posterior. Outra característica comum aos dois fluxos é que mais de $80 \%$ dos migrantes declararam estar em situação irregular nesse país.

TABELA 2

Distribuição dos Migrantes, por Cidade de Origem, segundo País de Destino na Primeira Viagem Governador Valadares e Criciúma - 1997-2001

\begin{tabular}{lrr}
\hline \multirow{2}{*}{ País de Destino } & \multicolumn{2}{c}{ Cidade de Origem } \\
\cline { 2 - 3 } & Governador Valadares & Criciúma \\
& 1997 & $\mathbf{2 0 0 1}$ \\
\hline Total (№s Absolutos) & $\mathbf{5 1 4}$ & $\mathbf{5 0 5}$ \\
Total (\%) & 100,00 & 100,00 \\
Estados Unidos & 85,60 & 60,00 \\
Canadá & 2,53 & 1,58 \\
Portugal & 2,33 & 10,89 \\
Itália & 0,78 & 14,26 \\
México & 0,19 & 4,55 \\
Outros & 8,57 & 8,72 \\
\hline
\end{tabular}

Fonte: Pesquisa sobre Migração Internacional de Brasileiros - 1997 e 2001. Nota: Os dados desta tabela são originados dos surveys realizados em Governador ValadaresMG (1997) e/ou em Criciúma-SC (2001), ambos coordenados pela Professora Dra. Teresa Sales e descritos neste artigo no item "Base de Dados".

Em alguns casos, o caminho até os Estados Unidos passa pelo México, ou seja, alguns brasileiros tentam o ingresso nesse país pela fronteira. Note-se que até 1997, ano da realização do survey em Governador Valadares, somente um habitante dessa cidade arriscou a travessia por essa fronteira. Os criciumenses, por participarem de um fluxo muito mais recente que o dos mineiros, sofrem mais com a freqüência de negativas aos pedidos de visto que vêm ocorrendo nos últimos anos por parte do consulado norteamericano no Brasil. Como conseqüência, verifica-se uma proporção muito maior de pessoas de Criciúma - 23 migrantes - seguindo para os Estados Unidos depois de passarem pelo México. Ao que indicam as mais recentes notícias veiculadas pela mídia, o fluxo de brasileiros que tentam a sorte por meio da travessia da fronteira MéxicoEstados Unidos tem aumentado continuamente.

\section{FORMAÇÃO DAS COMUNIDADES-FILHAS}

O que dificulta o reconhecimento preciso da distribuição de brasileiros nos Estados Unidos é a ausência de dados oficiais mais específicos sobre o destino dos emigrantes. A variável do Censo Demográfico brasileiro que registra o país de residência anterior, por exemplo, não é acompanhada de uma variável complementar para determinar em que estado e município daquele país vivia o migrante retornado. Por meio dos surveys utilizados neste trabalho, no entanto, podemos reconhecer o local de destino dos emigrantes em nível municipal.

O reconhecimento dos pontos de destino mais expressivos de um determinado fluxo permite uma caracterização adicional de sua dinâmica. Para citar alguns exemplos, tome-se a comunidade brasileira em San Francisco, nos Estados Unidos. Segundo Ribeiro (1999), dados do consulado brasileiro mostram que podemos encontrar migrantes de vários estados do Brasil, mas que a concentração de goianos é a expressão mais evidente; Oliveira (2003), em sua pesquisa de campo na Flórida, encontrou brasileiros originários predominantemente do Rio de Janeiro e de São Paulo. E os indivíduos que saem de Governador Valadares e Criciúma, onde se concentram? A Tabela 3 mostra o estado de destino desses brasileiros em sua primeira viagem aos EUA.

Os valadarenses concentram-se predominantemente em Massachusetts, Flórida, Nova Jersey e Nova York (Tabela 3), mas o primeiro estado é, sem dúvida, o principal destino, uma vez que agrega mais de $51 \%$ da população migrante. As pessoas originárias de Criciúma estão ainda mais concentradas em um único estado: algo em torno de $83 \%$ desses migrantes encontravam-se em Massachusetts, que é, também, a região que mais atrai valadarenses. $\mathrm{Na}$ verdade, o movimento de saída mais expressivo em Criciúma acontece mais de 10 anos depois do ápice verificado para o fluxo de Valadares. Dessa forma, podemos considerar que os criciumenses seguiram o caminho tra- 
çado pelos valadarenses, encontrando uma comunidade de brasileiros em estágio de expansão no território norteamericano.

TABELA 3

Distribuição dos Migrantes, por Município de Origem, segundo Estado de Destino na Primeira Viagem para os Estados Unidos Governador Valadares e Criciúma - 1997-2001

\begin{tabular}{lc}
\hline Estado de Destino & Governador Valadares (1997) \\
\hline Total (Nos Absolutos) & 403 \\
Total (\%) & 100,00 \\
Massachusetts & 51,86 \\
Flórida & 15,88 \\
Nova Jersey & 14,64 \\
Nova York & 11,66 \\
Outros & 5,96 \\
& \\
\hline Estado de Destino & Criciúma (2001) \\
\hline Total (Nos Absolutos) & 302 \\
Total (\%) & 100,00 \\
Massachusetts & 83,08 \\
Flórida & 5,38 \\
Connecticut & 3,85 \\
Texas & 1,92 \\
Outros & 5,77 \\
\hline
\end{tabular}

Fonte: Pesquisa sobre Migração Internacional de Brasileiros - 1997 e 2001. Nota: Os dados desta tabela são originados dos surveys realizados em Governador ValadaresMG (1997) e/ou em Criciúma-SC (2001), ambos coordenados pela Professora Dra. Teresa Sales e descritos neste artigo no item "Base de Dados".

Observando as cidades de destino com maior concentração de brasileiros (Tabela 4), destaca-se, em primeiro lugar, a cidade de Boston, que sozinha é responsável por mais de um terço das migrações de Valadares, e quase $60 \%$ do movimento de Criciúma. Para Valadares, em seguida vem a cidade de Nova York, com 9,40\%; Newark, com 5,48\% ; e Framingham, que faz parte da região metropolitana de Boston, com 4,96\%. Na Flórida, destacam-se as cidades de Pompano Beach, Deerfield Beach e Miami, porém todas com contingentes de valadarenses que representam apenas $3 \%$ dos migrantes. No caso de Criciúma, a segunda cidade de destino em importância é Somerville, que também está inserida na região metropolitana de Boston e que acrescenta quase $7 \%$ de criciumenses a essa área. Em síntese, Massachusetts e, principalmente, a região metropolitana de Boston são os locais que mais concentram os migrantes que partiram de Governador Valadares e de Criciúma.
TABELA 4

Distribuição dos Migrantes, por Município de Origem, segundo Cidade de Destino na Primeira Viagem para os Estados Unidos Governador Valadares e Criciúma - 1997-2001

\begin{tabular}{lc}
\hline Cidade de Destino & Governador Valadares (1997) \\
\hline Total (№s Absolutos) & 383 \\
Total (\%) & 100,00 \\
Boston & 37,60 \\
Nova York & 9,40 \\
Newark & 5,48 \\
Framingham & 4,96 \\
Outras & 42,56 \\
& \\
\hline Cidade de Destino & 292 \\
\hline Total (№s Absolutos) & Criciúma (2001) \\
Total (\%) & 100,00 \\
Boston & 58,90 \\
Somerville & 6,85 \\
Miami & 4,11 \\
Hartford & 3,42 \\
Outras & 26,72 \\
\hline Fonte: Pesquisa sobre Migração Internacional de Brasileiros - 1997 e 2001. \\
Nota: Os dados desta tabela são originados dos surveys realizados em Governador Valadares- \\
MG (1997) e/ou em Criciúma-SC (2001), ambos coordenados pela Professora Dra. Teresa \\
Sales e descritos neste artigo no item "Base de Dados". \\
\end{tabular}

O município de Framingham abriga uma das mais notáveis comunidades de imigrantes brasileiros, provenientes principalmente de Governador Valadares. Segundo Bicalho (1989), 87\% dos residentes em Framingham vieram do Vale do Rio Doce. Teresa Sales (1999, p. 47), que dedicou um capítulo especial à cidade norte-americana, relata o sentimento de identidade provocado pela predominância de brasileiros no local.

Ao sair novamente à rua, apesar do frio de outono daquele final de tarde apressado em escurecer mais cedo, me sinto brasileirinha da silva. Tão brasileira depois daquela coxinha de galinha e daquele suco de caju, que estranhei quando, na rua, me deparei com dois autênticos nativos conversando em inglês. ${ }^{3}$

Os valadarenses, portanto, formam comunidades importantes em quatro Estados dos EUA, mas a concentração de mais de $51 \%$ em Massachusetts evidencia este como o principal ponto de destino desses brasileiros. Essa informação também pode ser encontrada nos trabalhos de Sales (1999), Martes (1998) e Bicalho (1989), que pesquisaram sobre a origem dos imigrantes brasileiros nos 
Estados Unidos. Os criciumenses aparecem ainda mais concentrados em Massachusetts que os valadarenses, mas devido ao caráter extremamente recente desse fluxo, não encontramos referências a ele na literatura especializada.

Uma vez que a chegada dos brasileiros em território dos Estados Unidos acontece de forma contínua, podemos concluir que muitos migrantes seguiram paulatinamente para locais onde já se encontravam seus conterrâneos. Esse é um dos aspectos das redes sociais, que, segundo Sales (1999, p. 36)

contribuem não apenas para fornecer os referenciais do local de destino, como a acomodação inicial do imigrante e sua inserção no mercado de trabalho.

A Tabela 5 mostra o estado de destino do migrante valadarense em função do período da primeira viagem aos EUA. Os dados de Criciúma não podem ser apresentados dessa forma, pois o caráter recente desse movimento concentra mais de $50 \%$ dos migrantes nos três últimos anos antes da pesquisa, indicando que o ápice ainda não foi atingido, o que inviabiliza a desagregação da informação em períodos. O fluxo de Valadares, por outro lado, teve seu auge dez anos antes da pesquisa - o que permite classificar os períodos em: aceleração, ápice e desaceleração/ estabilização. Dessa forma, vemos que Massachusetts atraiu $45,1 \%$ dos migrantes que fizeram sua primeira viagem até 1986. Essa concentração aumentou para 50,6\% no segundo período, e cresceu ainda mais no período mais recente, chegando a contar com 58,5\% dos migrantes que saíram do Brasil entre os anos de 1990 e 1997. O fluxo que se direcionou para Nova Jersey teve um crescimento

\section{TABELA 5}

Distribuição dos Migrantes, por Período de Migração, segundo Estado de Destino na Primeira Viagem para os Estados Unidos Governador Valadares - 1967-1997

\begin{tabular}{lccc}
\hline \multirow{2}{*}{ Estado de Destino } & \multicolumn{3}{c}{ Período da Migração } \\
\cline { 2 - 4 } & 1967 a 1986 & 1987 a 1989 & 1990 a 1997 \\
\hline Total (№s Abs.) & 122 & 166 & 118 \\
Total (\%) & 100,0 & 100,0 & 100,0 \\
Massachusetts & 45,1 & 50,6 & 58,5 \\
Flórida & 18,9 & 13,9 & 12,7 \\
Nova Jersey & 12,3 & 16,3 & 14,4 \\
Nova York & 18,0 & 10,8 & 5,1 \\
Outros & 5,7 & 8,4 & 9,3 \\
\hline
\end{tabular}

Fonte: Pesquisa sobre Migração Internacional de Brasileiros - 1997.

Nota: Os dados desta tabela são originados do survey realizado em Governador ValadaresMG (1997), coordenado pela Professora Dra. Teresa Sales e descrito neste artigo no item "Base de Dados". tímido entre o primeiro e o último período de primeiras viagens, passando de $12,3 \%$ para $14,4 \%$. O que se verifica para os estados da Flórida e de Nova York é uma diminuição contínua da concentração de migrantes: com 18,9\% e $18,0 \%$, respectivamente, no primeiro período, a proporção de indivíduos que escolhem esses destinos em sua primeira viagem cai para $12,7 \%$ e $5,1 \%$ no último período.

O direcionamento de fluxos migratórios para pontos específicos de destino também foi estudado por Massey (1987). Esse autor descreve a formação de comunidades de migrantes, que ele chamou de "comunidades-filhas", como um processo intrínseco da migração. Os primeiros momentos da migração de mexicanos para os EUA comportavam uma diversidade muito maior de destinos do que nos períodos mais recentes. É um processo social que leva tempo para operar, de modo que as comunidades-filhas se desenvolvem mais devagar no início, aumentando o ritmo de crescimento quando um volume maior de migrantes passa a sustentar e alimentar as redes de forma mais consistente, fato que exerce um efeito magnético para as migrações subseqüentes. O Gráfico 1 reflete os dados da Tabela 5 e mostra a correspondência entre o processo migratório brasileiro em questão e as observações de Douglas Massey sobre a migração de mexicanos para os Estados Unidos.

O desenvolvimento de comunidades estabelecidas nos Estados Unidos é um passo crucial na maturação das redes migratórias. Quando alguns indivíduos se fixam em

\section{GRÁFICO 1}

Distribuição dos Migrantes, por Estado de Destino na Primeira Viagem para os Estados Unidos, segundo o Período de Migração Governador Valadares - 1967-1997

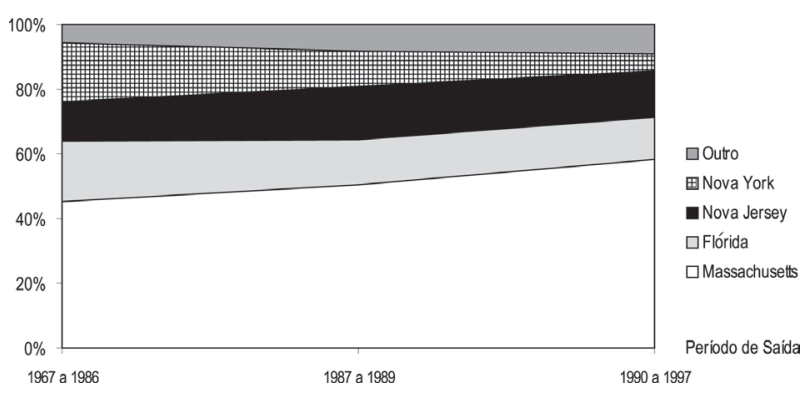

Fonte: Pesquisa sobre Migração Internacional de Brasileiros - 1997. Nota: Os dados desta tabela são originados do survey realizado em Governador ValadaresMG (1997), coordenado pela Professora Dra. Teresa Sales e descrito neste artigo no item "Base de Dados". 
determinados lugares, o processo migratório transformase, redirecionando os fluxos para regiões específicas (MASSEY, 1987). Visto desse modo, em todo local que se forma uma comunidade de imigrantes, a atração para novos migrantes parece um fato consumado. Mas não é isso o que pode ser observado no caso analisado. Enquanto, proporcionalmente ao total de pessoas em cada período, Massachusetts recebe cada vez mais indivíduos, decresce o número relativo de migrantes que se dirigem aos outros estados, com exceção de Nova Jersey, que mantém certa estabilidade.

O que ocorre é que não basta a aglomeração de pessoas no destino para que existam os benefícios que facilitam e estimulam o ingresso de pessoas num fluxo migratório. A formação de comunidades no destino é uma condição necessária, mas não suficiente. É preciso que se desenvolvam redes de relações confiáveis unindo origem e destino, nas quais o migrante em potencial ou o retornado possam apoiar-se. As diferenças verificadas na Tabela 3 podem ser explicadas em função desse argumento, ou seja, as regiões que mais atraem migrantes são as que melhor disponibilizam os recursos do capital social presentes nas redes migratórias.

\section{CAPITAL SOCIAL E DINÂMICA MIGRATÓRIA}

Uma vez que Massachusetts concentra mais de $83 \%$ dos emigrantes de Criciúma que se dirigem aos Estados Unidos, as variáveis de uso dos recursos do capital social não suportam a desagregação em função dos outros estados de destino. Foi possível, no entanto, separar os migrantes em "presentes/retornados" e "ausentes/exterior". Dessa forma, os resultados da Tabela 6 referem-se de modo quase integral aos criciumenses que decidiram viver em Massachusetts, e pretendem indicar a influência do uso dos recursos das redes sociais na manutenção do status de migrante.

De acordo com a Tabela 6, cerca de $56 \%$ dos criciumenses ausentes e $45 \%$ dos retornados recorreram à ajuda de terceiros para levantar os recursos financeiros necessários para realizar a empreitada; com relação à hospedagem, mais de $91 \%$ dos ausentes e cerca de $90 \%$ dos retornados solicitaram e receberam ajuda nesse quesito; a obtenção do primeiro emprego no destino foi atingida com a colaboração de parentes e amigos para mais de $86 \%$ dos ausentes e para mais de $85 \%$ dos retornados. Apesar de pequena, a diferença entre a proporção de utilização dos recursos do capital social entre retornados e ausentes pode ser verificada. Entre os que melhor se aproveitam desses recursos, esse fato poderia indicar maior propensão de manter-se no destino. As agências de recrutamento, por sua vez, não se configuram como uma fonte utilizada com freqüência para a obtenção desses mesmos recursos. De qualquer forma, podemos concluir que, para a maioria dos criciumenses, os recursos do capital social influenciam fortemente o processo de adaptação no destino - o qual, nesse caso, está cristalizado em Massachusetts.

Por outro lado, devido à maior diversidade dos locais de destino, os dados de Valadares comportam a apresentação dos recursos utilizados pelos migrantes desagregados por estado. As Tabelas 7 e 8 foram construídas associando os dados sobre quem o valadarense conhecia no destino e quem lhe forneceu ajuda financeira para chegar

TABELA 6

Distribuição das Fontes de Recursos para o Migrante em sua Primeira Viagem aos Estados Unidos, por Tipo de Recurso e Status Migratório Criciúma - 2001

\begin{tabular}{|c|c|c|c|c|c|c|}
\hline \multirow{2}{*}{ Fontes } & \multicolumn{2}{|c|}{ Recursos Financeiros } & \multicolumn{2}{|c|}{ Hospedagem } & \multicolumn{2}{|c|}{ Emprego } \\
\hline & Retornado & Ausente & Retornado & Ausente & Retornado & Ausente \\
\hline Total (№s Abs.) & 142 & 406 & 143 & 406 & 124 & 353 \\
\hline Total (\%) & 100,0 & 100,0 & 100,0 & 100,0 & 100,0 & 100,0 \\
\hline Próprios & 54,2 & 44,0 & 9,8 & 8,4 & 14,5 & 13,9 \\
\hline Parentes & 38,0 & 47,0 & 43,4 & 44,8 & 30,6 & 34,8 \\
\hline Amigos & 3,5 & 4,2 & 39,2 & 41,4 & 50,0 & 46,5 \\
\hline Ag. Recrutamento & - & 1,2 & 0,6 & 1,2 & - & 0,6 \\
\hline Outros & 4,3 & 3,6 & 7,0 & 4,2 & 4,9 & 4,2 \\
\hline
\end{tabular}

Fonte: Pesquisa sobre Migração Internacional de Brasileiros - 2001.

Nota: Os dados desta tabela são originados do survey realizado em Criciúma-SC (2001), coordenado pela Professora Dra. Teresa Sales e descrito neste artigo no item "Base de Dados". 
TABELA 7

Quem o Migrante Conhecia no Destino, segundo o Estado de Destino na Primeira Viagem aos Estados Unidos Governador Valadares - 1997

\begin{tabular}{lcccccc}
\hline \multirow{2}{*}{ Estado de Destino } & Total & \multicolumn{4}{c}{ Quem Conhecia no Destino } \\
\cline { 2 - 7 } & (№s Absolutos) & Parentes & Amigos & Ninguém & Outra & Total \\
\hline Massachusetts & 208 & 56,3 & 26,0 & 17,3 & 0,4 & 100,0 \\
Flórida & 61 & 50,9 & 21,3 & 27,8 & 0,0 & 100,0 \\
Nova Jersey & 59 & 64,4 & 15,3 & 20,3 & 0,0 & 100,0 \\
Nova York & 46 & 47,8 & 28,3 & 23,9 & 0,0 & 100,0 \\
Outro & 31 & 58,1 & 19,3 & 22,6 & 0,0 & 100,0 \\
\hline
\end{tabular}

Fonte: Pesquisa sobre Migração Internacional de Brasileiros - 1997.

Nota: Os dados desta tabela são originados do survey realizado em Governador Valadares-MG (1997), coordenado pela Professora Dra. Teresa Sales e descrito neste artigo no item "Base de Dados".

TABELA 8

Distribuição da Fonte de Recursos Financeiros para o Migrante, segundo o Estado de Destino na Primeira Viagem aos Estados Unidos Governador Valadares - 1997

\begin{tabular}{|c|c|c|c|c|c|c|c|}
\hline \multirow{2}{*}{ Estado de Destino } & \multirow{2}{*}{$\begin{array}{c}\text { Total } \\
\text { (№s Absolutos) }\end{array}$} & \multicolumn{6}{|c|}{ Fonte de Recursos Financeiros } \\
\hline & & Próprios & Parentes & Amigos & Ninguém & Outra & Total \\
\hline Massachusetts & 208 & 38,0 & 44,7 & 9,6 & 3,4 & 2,9 & 100,0 \\
\hline Flórida & 61 & 64,0 & 27,9 & 4,9 & 1,6 & 1,6 & 100,0 \\
\hline Nova Jersey & 59 & 52,5 & 39,0 & 6,8 & 1,7 & 0,0 & 100,0 \\
\hline Nova York & 46 & 45,7 & 32,6 & 10,9 & 8,7 & 2,2 & 100,0 \\
\hline Outro & 31 & 48,4 & 38,7 & 6,5 & 3,2 & 3,2 & 100,0 \\
\hline
\end{tabular}

Fonte: Pesquisa sobre Migração Internacional de Brasileiros - 1997.

Nota: Os dados desta tabela são originados do survey realizado em Governador Valadares-MG (1997), coordenado pela Professora Dra. Teresa Sales e descrito neste artigo no item "Base de Dados".

ao estado de destino na primeira viagem. Pudemos, então, retomar a hipótese inicial de que, em determinadas regiões, a atração exercida sobre os migrantes está diretamente relacionada à dinâmica de transferência dos benefícios próprios das redes migratórias.

As pessoas que chegaram a Massachusetts fizeram uso da melhor estrutura de relações dentre todos os estados de destino: somente $17,3 \%$ relataram não conhecer ninguém, enquanto que $56,3 \%$ tinham parentes no destino, e os amigos foram a principal referência para $26,0 \%$ desse grupo, conforme a Tabela 7. Os migrantes que se dirigiram para Flórida e Nova York apresentam o maior índice de pessoas sem nenhum conhecido nos Estados Unidos, com $27,8 \%$ e $23,9 \%$, respectivamente.

Conforme a Tabela 8 , os migrantes que não utilizaram fontes alternativas de recursos financeiros aparecem em maior quantidade nos Estados da Flórida, Nova Jersey e Nova York, com a proporção interna a cada grupo de
$64,0 \%, 52,5 \%$ e 45,7\%, respectivamente. Mais uma vez, pudemos destacar a presença e a importância das redes que ligam Governador Valadares a Massachusetts e dos recursos que por elas circulam. Apenas 38,0\% desse grupo contaram somente com recursos próprios, e a proporção daqueles que conseguiram ajuda financeira entre parentes atinge $44,7 \%$ - o maior índice da tabela para essa categoria.

A simples utilização de recursos financeiros de parentes ou amigos não significa necessariamente que essas pessoas ficaram em vantagem com relação àqueles que contaram somente com recursos próprios. Alguns têm maior poder econômico que outros, e essa pode ser a razão da não necessidade de empréstimos. Entretanto, o que se evidencia aqui é que existe, de fato, maior disponibilidade e uso de recursos, financeiros ou não, que justificam a maior concentração de migrantes em determinadas regiões. 


\section{CONCLUSÃO}

Um importante passo no amadurecimento das redes migratórias ocorre quando alguns migrantes começam a se estabelecer nos Estados Unidos. Quando as novidades positivas percorrem a região de origem, mais indivíduos se encorajam e seguem os passos dos pioneiros. A existência de regiões com concentração de parentes, amigos e conhecidos, por sua vez, acelera a expansão das redes, dando a elas uma forte base nos EUA. No entanto, os pontos de destino encontram-se em diferentes fases quanto à circulação dos recursos por meio das redes formadas em cada local. Observou-se que as redes sociais que ligam Governador Valadares e Criciúma a Massachusetts são as que melhor disponibilizam seus recursos, e que é essa região nos EUA que mais atrai os migrantes valadarenses e criciumenses. Como resultado, encontramos atualmente diversas instituições associativas para brasileiros em Massachusetts, evidenciando o desenvolvimento de uma comunidade bastante "visível", ao contrário do que Maxine Margolis encontrou em Nova York.

Como vimos, a migração de valadarenses e criciumenses para os Estados Unidos é fortemente baseada numa organização social que a apóia e sustenta. Massey (1987, p. 169) diz que

a migração internacional é um processo social organizado através de redes forjadas diariamente pelas conexões interpessoais que caracterizam todos os grupos humanos.

Percebemos a presença dessas conexões cotidianas transformadas no contexto das migrações e a ampliação do uso das redes com o passar dos anos. As redes migratórias tendem a se tornar auto-suficientes com o tempo, devido ao capital social que elas geram aos migrantes em potencial, pois ao mesmo tempo em que as redes se expandem, novas situações de oferta e demanda de recursos são reproduzidas e negociadas entre os novos migrantes. Contatos pessoais com parentes, amigos e conterrâneos dão aos migrantes o necessário acesso a emprego, hospedagem e assistência financeira no destino, canalizando pessoas que fazem parte de certos grupos sociais no Brasil para regiões específicas nos Estados Unidos. A confiança é - claramente - um elemento fundamental nesse processo. Afinal, os preciosos recursos que facilitam a inserção dos migrantes no país de destino e, conseqüentemente, o direcionamento desses migrantes para determinados locais, estão restritos a relacionamentos em que os atores envolvidos são pautados pelo reconhecimento do risco envolvido na transferência desses mesmos recursos - ou na ausência dele.

\section{NOTAS}

1. No caso das pesquisas realizadas, o erro máximo em Governador Valadares ficou em 5,0\% e em Criciúma ficou em 5,3\%; em ambas cidades o intervalo de confiança foi de $95 \%$.

2. Este termo é utilizado para designar o descendente de japonês que migra para trabalhar no Japão.

3. Este é um depoimento da própria autora, em seu livro Brasileiros Longe de Casa (SALES, 1999).

\section{REFERÊNCIAS BIBLIOGRÁFICAS}

ASSIS, G.O. De Criciúma para o mundo: rearranjos familiares e de gênero nas vivências dos novos migrantes brasileiros. Tese (Doutorado em Ciências Sociais) - IFCH-Unicamp, Campinas, 2004.

Estar aqui..., estar lá... uma cartografia da emigração valadarense para os EUA. In: REIS, R.R.; SALES, T. (Org.). Cenas do Brasil migrante. São Paulo: Boitempo, 1999.

BICALHO, J.V. Yes, eu sou brazuca. Governador Valadares: Ibituruna, 1989.

BOURDIEU, P.; WACQUANT, L. An Invitation to Reflexive Sociology. Chicago: University of Chicago Press, 1992.

BOYD, M. Family and personal networks in international migration: recente developments and new agendas. International Migration Review, New York, CMS of NY, v. XXIII, n. 3, 1989.

CARVALHO, J.AM. O saldo dos fluxos internacionais do Brasil na década de 80: uma tentativa de estimação. In: PATARRA, N. (Org.). Migrações internacionais. São Paulo: Oficina Editorial, 1996. v. 2.

CASTLES, S.; MILLER, M.J. The age of migration: International population movements in the modern world. Hampshire London: Macmillan, 1995.

COLEMAN, J.S. Foundations of Social Theory. Cambridge, Mass: The Belknap Press of Harvard University Press, 1990.

DEBIAGGI, S.D. Famílias brasileiras em um novo contexto cultural. In: MARTES, C.; FLEISCHER, S. (Org.). Fronteiras Cruzadas. São Paulo: Paz e Terra, 2003.

DEGENNE, A.; FORSÉ, M. Introducing Social Networks. GrãBretanha: Sage Publications, 1999.

DINERMAN, I.R. Patterns of adaptation among households of U.S. - Bound migrants from Michoacan, Mexico. International Migration Review, New York, CMS of NY, v. XII, n. 4, 1978.

MARGOLIS, M. Little Brazil: imigrantes brasileiros em Nova York. Campinas: Papirus, 1994.

MARTES, A.C.B. Brasileiros nos Estados Unidos: Imigrantes brasileiros em Massachusetts. São Paulo: Paz e Terra, 1998.

MASSEY, D.S. et al. Return to aztlan. Los Angeles: University of California Press, 1987. 
MENJIVAR, C. Immigrant Kinship Networks and the Impact of the Receiving Context: Salvadorans. Social Problems, San Francisco, University of California Press, v. 44, n. 1, Feb. 1997.

OLIVEIRA, A.C. O caminho sem volta: Classe social e etnicidade entre os brasileiros na Flórida. In: MARTES, A.C.B; FLEISCHER, S. (Org.). Fronteiras Cruzadas. São Paulo. Paz e Terra, 2003.

PALAU, T. Brasiguaios. In: CASTRO, M. (Org.). Migrações internacionais: contribuições para políticas. Brasília, DF: CNPD, 2001.

PORTES, A. et al. Transnational Entrepeneurs: An Alternative Form of Immigrant Economic Adaptation. American Sociological Review, Washington, DC, University of Pennsylvania Press, v. 67, Apr. 2002.

RIBEIRO, G.L. O que faz o Brasil, Brazil: jogos identitários em São Francisco. In: REIS, R.R.; SALES, T. (Org.). Cenas do Brasil migrante. São Paulo: Boitempo, 1999.

SALES, T. Brasileiros longe de casa. São Paulo: Cortez, 1999.

SASAKI, E.M. Movimento dekassegui: a experiência migratória e identitária dos brasileiros descendentes de japoneses no Japão. In: REIS, R.R.; SALES, T. Cenas do Brasil migrante. São Paulo: Boitempo, 1999.
SCUDELER, V. Imigrantes valadarenses no mercado de trabalho dos EUA. In: REIS, R.R.; SALES, T. (Org.). Cenas do Brasil migrante. São Paulo: Editora Boitempo, 1999.

SOARES, W. Da metáfora à sustância: redes sociais, redes migratórias e migração nacional e internacional em Valadares e Ipatinga. Tese (Doutorado em Demografia) - Universidade Federal de Minas Gerais, Belo Horizonte, 2002. imobiliária na economia valadarense. Dissertação (Mestrado) Instituto de Pesquisa e Planejamento Urbano e Regional/Universidade Federal do Rio de Janeiro, Rio de Janeiro, 1995.

UNITED STATES BUREAU OF THE CENSUS. Current Population Survey. Washington, DC: Department of Commerce, 2000.

Wilson Fusco: Doutor em Demografia pelo Nepo/IFCH/Unicamp (Campinas)(wfusco@nepo.unicamp.br).

Artigo recebido em 5 de maio de 2005 . Aprovado em 29 de agosto de 2005. 